\section{Fabienne Lamballe}

\section{ADRESSE}

F. Lamballe: chargée de recherche à l'Inserm Cnrs UMR 146, Institut Curie-Biologie, centre universitaire, laboratoire 110,91405 Orsay Cedex, France.

$m / s n^{\circ} 8$, vol. 11 , août 95

\title{
Les récepteurs tyrosine kinases Trk : récepteurs de forte affinité des neurotrophines
}

Les neurotrophines de la famille du NGF (nerve growth factor) sont impliquées dans la régulation de la différenciation, la croissance et la survie des neurones. Leurs effets sont assurés par les récepteurs tyrosine kinases de la famille Trk. Toutefois, les neurotrophines se lient également à un autre récepteur, la $\mathrm{p} 75^{\mathrm{LNGFR}}$, dont le rôle est controversé. Les récepteurs Trk sont exprimés préférentiellement dans le système nerveux. Malgré leur similitude avec les autres récepteurs tyrosine kinases, ils s'en distinguent par le fait qu'ils possèdent de multiples isoformes (isoformes non catalytiques et isoformes possédant des insertions de taille variable dans le domaine catalytique tyrosine kinase) qui ont vraisemblablement des rôles physiologiques particuliers. L'analyse de souris porteuses de mutations nulles pour les neurotrophines et leurs récepteurs devrait nous apporter une meilleure compréhension de la fonction de ces facteurs et leur possible utilisation thérapeutique dans des maladies neurodégénératives variées.

e système nerveux des mammifères est probablement le système biologique le plus évolué à l'heure actuelle et présente un très grand degré de complexité. Cela explique sans doute le nombre croissant d'équipes qui étudient le développement embryonnaire et la différenciation des cellules neuronales et gliales. Une caractéristique paradoxale du développement du système nerveux chez les vertébrés est la mort cellulaire [1]. En fait, ce phénomène existe dans de nombreux types de cellules nerveuses et cela pendant une période restreinte du développement, très peu de temps après que les neurones ont innervé leurs cibles.

Il y a environ quarante ans, Levi-Montalcini, Cohen et Hamburger proposèrent que les cellules cibles régleraient la densité de leur propre innervation par la présence de quantités limitantes de facteurs de survie des neurones. Cette hypothèse fut alors renforcée par l'identification de la première neurotrophine, le NGF (nerve growth factor) [2]. 


\section{RÉEÉRENCES}

1. Martinou J. La mort cellulaire programmée dans le système nerveux. médecine/sciences $1995 ; 11: 367-73$.

2. Levi-Montalcini R. The nerve growth factor 35 years later. Science 1987; 237: 1154 62 .

3. Hamburger V. The history of the discovery of the nerve growth factor. I Neurobiol $1993 ; 24: 893-7$.

4. Ebendal T. Function and evolution in the NGF family and its receptors. I Neurosci Res $1992 ; 32$; $461-70$.

5. Thoenen $\mathrm{H}$. The changing scene of neurotrophic factor. Trends Neurosci 1991; 14 : $165-70$

6. Barbacid M. Nerve growth factor: a tale of two receptors. Oncogene $1993 ; 8: 2033-42$.

7. Lee KF, Li E, Huber LJ, Landis SC, Sharpe AH, Chao MV, Yaenisch R. Targeted mutation of the gene encoding the low affinity NGF receptor p75 leads to deficits in the peripheral sensory nervous system. Cell 1992 ; 69 : $737-49$

8. Rabizadeh S, Oh J, Zhong LT, Yang J, Bitler CM, Butcher LL, Bredesen DE. Induction of apoptosis by the low-affinity NGF receptor. Science 1993 ; 261 : 345-8.

9. Martin-Zanca D, Hughes S, Barbacid M. A human oncogene formed by the fusion of truncated tropomyosin and protein tyrosine kinase sequences. Nature 1986 ; 319: 743-8.

10. Martin-Zanca D, Oskam R, Mitra G, Barbacid M. Molecular and biochemical characterization of the human trk proto-oncogene. Mol Cell Biol 1989; 9 : 24-33.

11. Schneider R, Schweiger M. A novel modular mosaic of cell adhesion motifs in the extracellular domains of the neurogenic irk and $t$ rkB tyrosine kinase receptors. Oncogene 1991 ; 6 : 1807-11.

12. Ollendorff V, Noguchi T, Birnbaum D. Des protéines à motifs riches en leucine définissent une cinquième famille de molécules d'adhérence. médecine/sciences $1993 ; 9$ :

\section{Les neurotrophines}

Le NGF a été identifié comme une substance diffusible capable de stimuler la différenciation et la survie des neurones sympathiques et sensoriels $[2,3]$. Pendant la dernière décennie, de nombreuses études ont montré que le NGF peut également jouer un rôle de survie et de maintien de la fonction des neurones cholinergiques du cerveau antérieur basal (neurones qui dégénèrent dans le cas de la maladie d'Alzheimer) [4]. Le NGF semble donc jouer un rôle dans la survie de certaines populations bien spécifiques de neurones, à la fois dans le système nerveux central et le système nerveux périphérique.

En 1982, un second facteur neurotrophique, BDNF (brain-derived neurotrophic factor), fut purifié par Barde et al. [5]. Le clonage moléculaire du gène codant pour BDNF, en 1989, révéla que ce facteur neurotrophique était fortement apparenté au NGF $(\mathrm{m} / \mathrm{s}$ $n^{\circ} 7$, vol. $6, p$. 700). Cette observation conduisit rapidement, grâce à la technique d'amplification de l'ADN par réaction de polymérisation en chaîne (PCR), au clonage de la neurotrophine-3 (NT-3) et de la neurotrophine-4 (NT-4, aussi appelée NT-4/5 ou NT-5) [5]. Ces facteurs neurotrophiques sont synthétisés sous forme de précurseurs polypeptidiques qui subissent une coupure protéolytique spécifique produisant des neurotrophines mûres. Ces molécules sont des homodimères de 115 à 130 acides aminés et présentent environ $50 \%$ d'identité de séquence [5]. Des études in vitro montrent que chaque neurotrophine induit la survie de certaines populations neuronales spécifiques. Toutefois, certains neurones peuvent être maintenus en survie par plus d'un facteur neurotrophique [5]. Les effets de ces facteurs de survie semblent être essentiels pour le développement normal et le maintien du système nerveux, mais également pour sa capacité à répondre en cas de lésion.

\section{Les récepteurs des neurotrophines}

Les neurotrophines se lient à deux catégories de protéines transmembranaires, la p75 ${ }^{\mathrm{INC} \text { NGR }}$ (aussi appelée récepteur de faible affinité pour le
NGF, LNGFR pour low affinity NGF receptor) et les membres de la famille des récepteurs tyrosine kinases Trk (m/s $n^{\circ} 6$, vol. 7, p. 620).

Le LNGFR est constitué d'une seule chaîne polypeptidique transmembranaire de $75 \mathrm{kDa}$. Son domaine extracellulaire possède quatre régions riches en résidus cystéine, caractéristiques d'une famille de récepteurs de surface qui comprend, entre autres, les récepteurs de type I et II du TNF (tumor necrosis factor), les antigènes de surface des lymphocytes CD30 et CD40 et l'antigène de surface Fas (qui semble être impliqué dans les phénomènes d'apoptose) $\left(m / s \quad n^{\circ} 2\right.$, vol. 10, p. 234) [1]. Cette région riche en résidus cystéine est responsable de la reconnaissance du ligand [6]. La courte région cytoplasmique, quant à elle, ne présente aucun motif particulier ni aucune analogie de séquence avec d'autres molécules connues. La fonction de ce domaine est, à l'heure actuelle, inconnue [6].

La $p 75^{\text {LNGFR }}$ se lie aux quatre neurotrophines de la famille du NGF avec une faible affinité, de l'ordre de la nanomole. Le rôle de ce récepteur reste obscur $\left(\mathrm{m} / \mathrm{s} n^{\circ} 10\right.$, vol. 10, p. 1049) [6]. Toutefois, il semble important pour le développement du système nerveux car des souris porteuses de mutations nulles du gène codant pour la $\mathrm{p} 75^{\text {L.NGFR }}$ présentent un déficit au niveau du système nerveux périphérique sensoriel qui se traduit par une innervation cutanée réduite et une sensibilité réduite à la douleur [7]. De plus, il a été récemment montré que ce récepteur induit l'apoptose de cellules neuronales lorsqu'il n'est pas lié au NGF [8] Les récepteurs tyrosine kinases de la famille Trk lient les neurotrophines avec des affinités plus fortes que celles de la p $75^{\text {I.NGir }}$ (de l'ordre de la picomole) et sont essentiels pour les réponses fonctionnelles. Ces protéines ont une structure en trois domaines qui comprend une région extracellulaire, un domaine transmembranaire et une région cytoplasmique. La région extracellulaire des récepteurs tyrosine kinases comprend certaines caractéristiques qui permettent de distinguer les différentes sous-classes de cette famille (figure 1). Les motifs identifiés à ce jour sont des régions riches en résidus cystéine ou leucine, des 


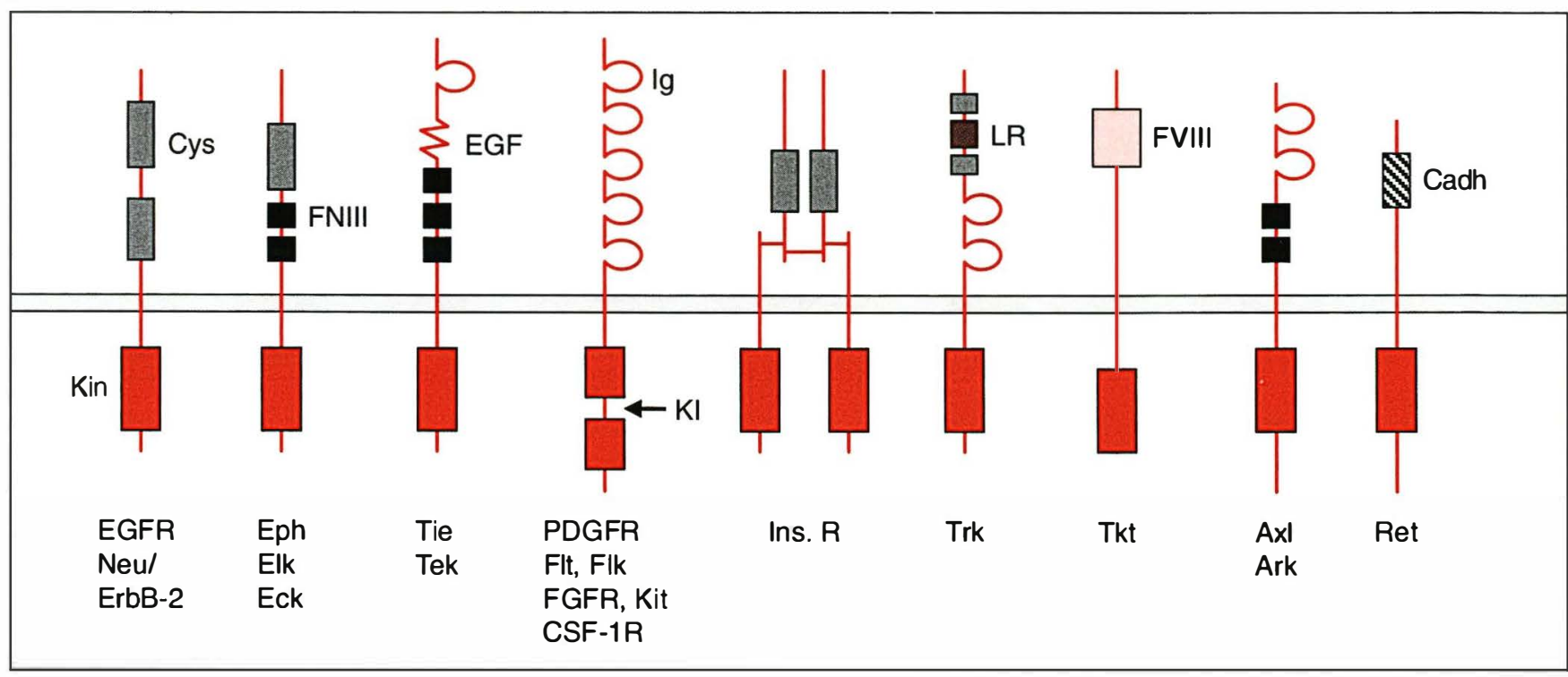

Figure 1. Les différentes sous-classes de récepteurs tyrosine kinases. Les différents récepteurs sont représentés avec leur domaine intracellulaire (domaine catalytique tyrosine kinase [Kin] et l'insertion située dans le domaine kinase [KI]) et les différents motifs structuraux de la région extracellulaire. Cys: région riche en résidus cystéine; FNIII: répétition de type fibronectine III; EGF: répétition de type EGF (epidermal growth factor); Ig: région présentant une homologie de structure avec celle des immunoglobulines; LR: région riche en résidus leucine; FVIII: région homologue aux unités $C 1$ et $C 2$ de la région carboxy-terminale du facteur VIII de coagulation; Cadh: motif de type cadhérine; Ins.R: récepteur de l'insuline. EGFR, PGDFR, CSF-1R: récepteurs de l'EGF, du PGDF, du FGF et du CSF-1.

domaines dont la structure présente une certaine analogie avec celle des immunoglobulines, des régions homologues des unités $\mathrm{Cl}$ et $\mathrm{C} 2$ de la partie carboxy-terminale du facteur VIII de coagulation et enfin des répétitions de type fibronectine III, cadhérine ou de type EGF. La région intracellulaire des récepteurs tyrosine kinases comprend le domaine catalytique de la molécule. Dans de nombreux cas, le mécanisme biochimique par lequel les récepteurs tyrosine kinases transmettent les signaux à travers la membrane comporte plusieurs étapes : tout d'abord, la dimérisation du récepteur induite par fixation du ligand; une fois le complexe dimérique formé, le récepteur s'autophosphoryle. Cette autophosphorylation entraîne ensuite la phosphorylation de substrats cytosoliques ainsi qu'une association avec d'autres molécules impliquées dans les effets pléiotropiques de la transmission du signal.

Le prototype de la famille des récepteurs Trk est le produit du gène trkA initialement appelé trk (pour tropomyosin receptor kinase). trkA a été iden-

$\mathrm{m} / \mathrm{s} n^{\circ} 8$, vol. 11 , août 95 tifié comme gène transformant dans une biopsie de carcinome du côlon humain $\left(m / s n^{\circ} 6\right.$, vol. $7, p$. 620). Ce nouvel oncogène était activé par réarrangement somatique entre des séquences codant pour les domaines transmembranaire et tyrosine kinase de TrkA et des séquences du gène de la tropomyosine non musculaire [9]. Le proto-oncogène trkA code pour une protéine transmembranaire de 790 acides aminés qui possède les caractéristiques de récepteurs tyrosine kinases [10]. Le domaine extracellulaire ne présente aucune analogie avec celui des autres récepteurs de facteur de croissance. En fait, le produit du proto-oncogène trkA possède une succession de motifs dans le domaine extracellulaire qui incluent une région riche en résidus leucine (LR) entourées de résidus cystéine (Cys), suivie de deux domaines présentant une analogie de structure avec celle des immunoglobulines (Ig) et qui pourraient être responsables de l'interaction avec le ligand (figure 2). La région riche en résidus leucine constitue une caractéristique de la super-famille des protéines
LRM (leucine-rich motifs) qui comprend des protéines de fonctions diverses telles que les protéines d'adhérence cellulaire et des composants de la matrice extracellulaire [11, 12]. Des études réalisées par hybridation in situ ont montré que l'expression du proto-oncogène trkA est très restreinte puisque les transcrits n'ont pu être détectés que dans les ganglions de la racine dorsale et certains ganglions crâniaux sensoriels dérivés de la crête neurale (ganglions des nerfs trijumeaux, supérieurs et jugulaires) [13].

Outre le gène trkA, trois loci désignés trkB, $t r k \mathrm{C}$ et $t r k \mathrm{E}$ ont été identifiés chez les mammifères. Un cinquième membre de la famille a, quant à lui, été identifié chez la drosophile et appelé Dtrk. L'identification de Dtrk suggère que la famille des récepteurs Trk a évolué à partir d'un gène ancestral commun ayant existé avant la séparation phylogénique entre les arthropodes et les chordés, il y a environ 800 millions d'années.

Les loci trk $\mathrm{B}$ et $t r k \mathrm{C}$ ont été identifiés par criblage de banques d'ADNc de cerveau avec une sonde correspon- 


\section{RÉFÉRENCES}

13. Martin-Zanca D, Barbacid M, Parada LF. Expression of the trk proto-oncogene is restricted to the sensory cranial and spinal ganglia of neural crest origin in mouse development. Genes Dev 1990; 4: 683-94.

14. Klein R, Parada LF, Coulier F, Barbacid M. $t r k B$, a novel tyrosine protein kinase receptor expressed during mouse neural receptor expressed during mouse neur
development. EMBO J $1989 ; 8: 3701-9$.

15. Lamballe F, Klein R, Barbacid M. $t r k \mathrm{C}$, a new member of the trk family of tyrosine protein kinases, is a receptor for neurotrophin-3. Cell 1991; 66: $967-79$.

16. Klein R, Conway D, Parada LF, Barbacid $\mathrm{M}$. The $t r k \mathrm{~B}$ tyrosine protein kinase gene codes for a second neurogenic receptor that lacks the catalytic kinase domain. Cell $1990 ; 61: 647-56$.

17. Klein R, Martin-Zanca D, Barbacid M, Parada LF. Expression of the tyrosine kinase receptor gene trkB is confined to the murine embryonic and adult nervous system. Development 1990; 109: 845-50.

18. Pulido D, Campuzano S, Koda T, Modolell J, Barbacid M. Dtrk, a Drosophila gene related to the trk family of neurotrophin receptors, encodes a novel class of neural cell adhesion molecule. $E M B O J 1992 ; 11$ : 391-404.

19. Di Marco E, Cutul N, Guerra L, Cancedda R, De Luca M. Molecular cloning of $t r k \mathrm{E}$, a novel trk-related putative tyrosine kinase receptor isolated from normal human keratinocytes and widely expressed by normal human tissues. I Biol Chem 1993; 268 : 24290-5.

20. Di Marco E, Mathor M, Bondanza S, Cutuli N, Marcisio PC, Cancedda R, De Luca M. Nerve growth factor binds to normal human keratinocytes through high and low affinity receptors and stimulates their growth by a novel autocrine loop. J Biol Chem 1993; 268: 22838-46.

21. Barbacid $M$. The Trk family of neurotrophin receptors: molecular characterization and oncogenic activation in human tumors. In: Levine AJ, Schmidek HH, eds. Molecular genetics of nervous system tumors.

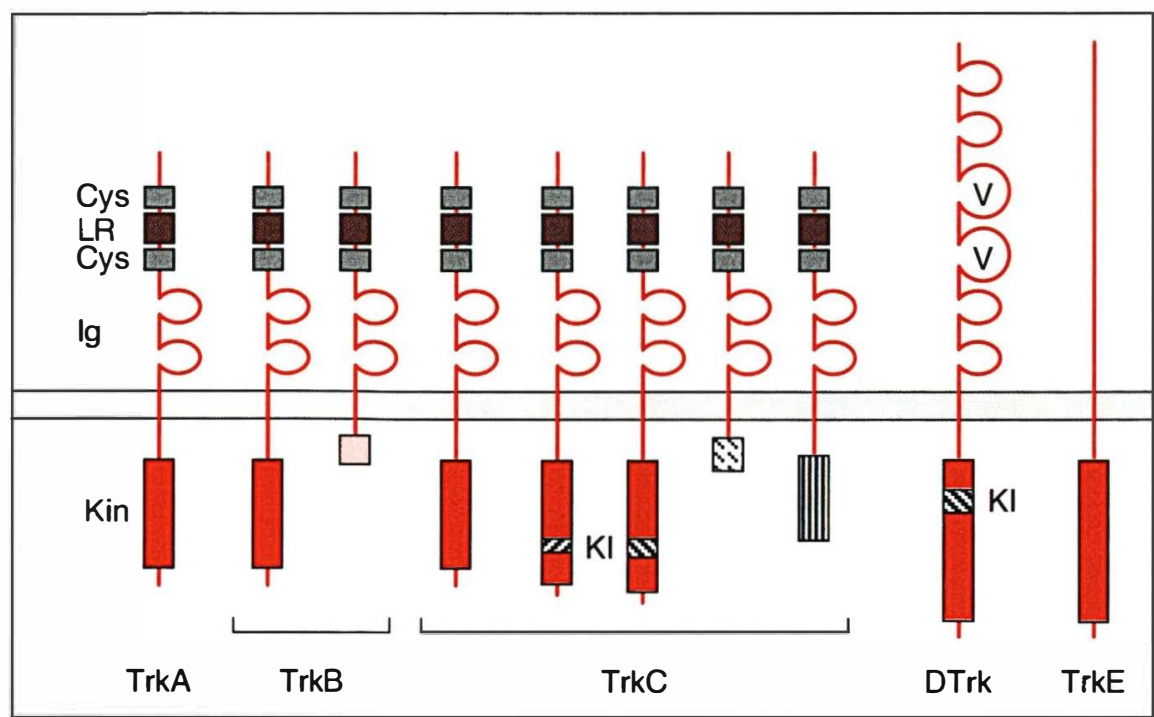

Figure 2. Les membres de la famille des récepteurs tyrosine kinases Trk. Le domaine extracellulaire des récepteurs TrkA, TrkB et TrkC possède une succession de motifs qui incluent une région riche en résidus leucine $(L R)$ entourés de résidus cystéine (Cys), suivie de deux domaines présentant une homologie de structure avec celle des immunoglobulines (Ig). Le récepteur DTrk présente six motifs homologues à la structure des immunoglobulines dont deux sont de type $V$. Le domaine extracellulaire de TrkE ne posssède aucune homologie significative avec aucune autre protéine connue. Les loci trkB et trkC codent pour plusieurs isoformes. Les rectangles rouges représentent les domaines catalytiques tyrosine kinases (Kin) dont certains comportent des insertions (KI, rectangles hachurés). Les régions carboxy-terminales des isoformes non catalytiques codées par les loci trkB et trkC sont représentées par les rectangles pointillés.

dant au domaine tyrosine kinase du proto-oncogène trkA $[14,15]$. Le locus trkB présente un profil transcriptionnel complexe (au moins six transcrits différents) et code pour au moins deux classes de récepteurs engendrés par épissage alternatif [16] (figure 2). Un des produits du gène, gpl $45^{1 \mathrm{rk}}$, est une protéine fortement glycosylée de $145 \mathrm{kDa}$ et possède une activité tyrosine kinase. Toutefois, trkB code également pour une deuxième glycoprotéine dépourvue de domaine catalytique tyrosine

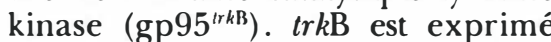
dans le système nerveux central et le système nerveux périphérique au cours du développement embryonnaire de la souris [14, 17]. Chez la souris adulte, trkB est préférentiellement exprimé dans le cerveau; toutefois, des transcrits $t r k \mathrm{~B}$ ont également été détectés dans le poumon, le muscle et les ovaires. Dans le cerveau de souris adultes, des études réalisées par hybridation in situ utilisant des sondes spécifiques pour les transcrits codant pour le récepteur catalytique $\left(\mathrm{gpl} 45^{\text {rkkB}}\right)$ ou le récepteur non catalytique ( $\left.\mathrm{gp} 95^{\text {rkk }}\right)$ ont montré un profil d'expression très particulier. Alors que les transcrits codant pour gp $145^{\prime \prime k B}$ sont présents dans le cortex cérébral et les cellules pyramidales de l'hippocampe, les ARN messagers codant pour le récepteur non catalytique gp95 ${ }^{t / k \mathrm{~B}}$ sont détectés dans le plexus choroïde et au niveau des cellules épendymaires des ventricules [16]. L'analyse du locus trkC a révélé un degré de complexité encore plus élevé que celui du locus trkB. En effet, ce gène code pour de multiples protéines isoformes, engendrées par épissage alternatif, dont le profil d'expression ainsi que les propriétés biochimiques et biologiques seront discutés ultérieurement.

DTrk, l'homologue des récepteurs Trk identifié chez la drosophile, pos- 


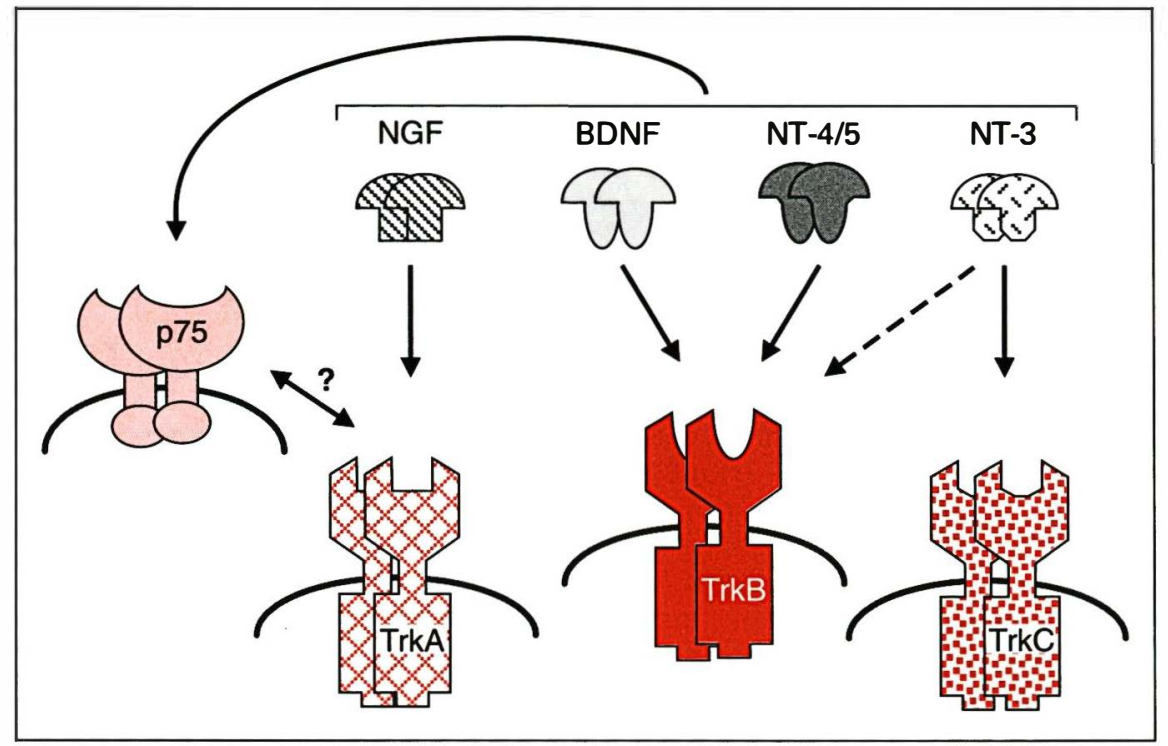

Figure 3. Interactions entre les neurotrophines et leurs récepteurs dans le système nerveux chez les mammifères. Les neurotrophines, sous forme de complexe homodimérique, activent leurs récepteurs respectifs de la famille Trk par induction de la dimérisation de ces récepteurs. Alors que TrkA est activé spécifiquement par le NGF, et TrkC par NT-3, TrkB peut être activé par BDNF et NT-4/5, et même par NT-3 dans certains cas. Toutes les neurotrophines se lient à la p75 LNGFR. Toutefois, la signification et la possibilité d'une association p $75^{L N G F R} / T r k$ sont très controversées.

sède dans sa région extracellulaire six domaines présentant une analogie de structure avec celle des immunoglobulines. Le domaine intracellulaire tyrosine kinase contient une insertion de 60 acides aminés qui créent un second site possible de fixation de l'ATP (figure 2). Des études récentes ont montré que la protéine DTrk est exprimée de manière dynamique dans le système nerveux de la drosophile au cours de l'embryogenèse. En outre, elle est impliquée dans les mécanismes d'adhérence cellulaire in vitro, processus qui conduit à la stimulation de son activité tyrosine kinase [18].

Enfin, le cinquième membre de la famille, $t r k \mathrm{E}$, a été récemment identifié dans des kératinocytes humains [19]. Toutefois, bien que la protéine TrkE appartienne à la famille des récepteurs Trk, son domaine extracellulaire ne possède pas d'analogie significative avec d'autres protéines

connues. En fait, l'appartenance à la famille Trk est fondée sur le pourcentage d'analogie de séquence et la présence de caractéristiques des récepteurs Trk localisées dans le domaine intracellulaire $[19,20]$. Il est à noter que trkE est le premier membre de cette famille de gènes exprimé abondamment en dehors du système nerveux $[19,20]$.

\section{Interactions entre les neurotrophines et leurs récepteurs}

L'expansion de la famille des neurotrophines et la caractérisation des récepteurs Trk a entraîné des désaccords concernant les possibilités d'interactions entre les neurotrophines et leurs récepteurs. Aussi, des expériences d'expression ectopique des récepteurs dans les fibroblastes ont été utiles pour établir les relations neurotrophines-récepteurs. De telles expériences ont montré que le NGF active le récepteur TrkA, que BDNF et NT-4/5 se lient et activent TrkB et enfin que TrkC est un récepteur à forte affinité pour NT-3 (figure 3) [15, 21, 22]. Toutef ois, la neurotrophine-3 semble également capable d'activer le récepteur TrkB, mais elle s'avère moins efficace que BDNF [21, 23]. De plus, les réponses engendrées semblent varier en fonction du type cellulaire dans lequel TrkB est exprimé. En effet, NT-3 engendre une réponse plus efficace dans des cellules fibroblastiques NIH3T3 que dans les cellules PC12 (dérivées de la crête neurale, capables de se différencier et ainsi de former des neurites) qui présentent le récepteur TrkB. Ces différences, inexpliquées à l'heure actuelle, pourraient impliquer des molécules accessoires (telles que la $\mathrm{p} 75^{\mathrm{LNGFR}}$ ) dont l'expression est restreinte aux cellules de type neuro-ectodermique.

Enfin, une question importante concerne le rôle de la $\mathrm{p} 75^{\mathrm{LNGFR}}$ qui se lie avec des affinités comparables à tous les membres de la famille des neurotrophines. Cette molécule estelle nécessaire pour convertir les récepteurs Trk en récepteurs de très forte affinité ? Cette question est de la plus grande importance parce qu'une telle conversion déterminerait la concentration à laquelle les neurotrophines exerceraient leur action.

\section{La transmission du signal déclenché par les récepteurs Trk}

La plupart des études sur la caractérisation des voies de signalisation à partir des récepteurs Trk ont été réalisées avec les fibroblastes NIH3T3 ou les cellules PC12. La caractérisation de ces voies dans des cellules primaires de neurones est bien moins avancée. Comme mentionné précédemment, pour d'autres récepteurs tyrosine kinases, la dimérisation des récepteurs Trk et la trans-phosphorylation sur résidus tyrosine sont des événements nécessaires pour assurer la réponse aux neurotrophines. Les résidus tyrosine phosphorylés servent alors de site d'ancrage à des protéines contenant des domaines $\mathrm{SH} 2$ (src-homology 2). Deux classes de molécules participent à cette cascade d'événements transductionnels : les 


\section{RÉFÉRENCES}

22. Meakin SO, Shooter EM. The nerve growth factor family of receptors. Trends Neurosci 1992 ; 15 : 323-31.

23. Ip NY, Stitt TN, Tapley P, Klein R, Glass D], Fandl J, Greene LA, Barbacid M, Yancopoulos GD. Similarities and differences in the way neurotrophins interact with the Trk receptors in neuronal and nonneuronal cells. Neuron 1993; 10: 137-49.

24. Kaplan DR, Stephens RM. Neurotrophin signal transduction by the Trk receptor. I Neurobiol 1994 ; 25 ; 1404-17.

25. Chardin P. Domaines SH2 et SH3: un nouveau paradigme pour la transmission du signal. médecine/sciences $1994 ; 10: 709-12$

26. Chardin P. Protéines Ras et transmission des signaux mitogènes. médecine/sciences $1994 ; 10: 657-64$.

27. Kahn A. La transmission du signal en amont et en aval de Ras. médecine/sciences $1992 ; 8: 1097-9$

28. Loeb DM, Tsao H, Cobb MH, Greene LA. NGF and other growth factors induce an association between ERK1 and the NGF receptor, gpl40 prototrk. Neuron 1992; 9 : 1053-65.

29. Rabin SJ, Cleghon V, Kaplan DR. SNT, a differentiation-specific target of neurotrophic factor-induced tyrosine kinase activity in neurons and PC12 cells. Mol Cell Biol $1993 ; 13: 2203-13$

30. Stephens RM, Loeb DM, Copeland TD, Pawson T, Greene LA, Kaplan DR. Trk receptors use redundant signal transduction pathways involving SHC and PLC- $\gamma 1$ to mediate NGF responses. Neuron $1994 ; 12$ : 691-705.

31. Traverse S, Gomez N, Paterson H, Marshall $C$, Cohen $P$. Sustained activation of the mitogen-activated protein (MAP) kinase cascade may be required for differentiation of PC12 cells. Comparison of the effects of nerve growth factor and epidermal growth factor. Biochem J 1992; 288: 351-5.

32. Tessarollo L, Tsoulfas $\mathbf{P}$, Martin-Zanca D, Gilbert DJ, Jenkins NA, Copeland NG, Parada LF. trkC, a receptor for neurotrophin-3, is widely expressed in the developing nervous system and in non-neuronal tissues. Development 1993; 118: 463-75.

33. Merlio JP, Ernfors P, Jaber M, Persson H. Molecular cloning of rat $t r k \mathrm{C}$ and distribution of cells expressing messenger RNAs for members of the $t r k$ family in the rat central nervous system. Neuroscience $1992 ; 51$. enzymes et les adaptateurs. Les enzymes sont phosphorylées sur résidus tyrosine par le récepteur tyrosine kinase, ce qui conduit à leur activation. Les adaptateurs, quant à eux, ne sont pas toujours phosphorylés. Leur rôle est de faciliter l'interaction entre différentes molécules de signalisation en les rapprochant au niveau de la membrane plasmique.

La transmission du signal déclenché par l'activation du domaine catalytique tyrosine kinase a surtout été étudiée pour le récepteur TrkA et le NGF [24]. A l'heure actuelle, les sites de liaison pour trois protéines cytoplasmiques ont été identifiés sur le récepteur TrkA. La protéine Shc se lie au résidu tyrosine juxtamembranaire Y490, la sous-unité régulatrice p85 de la phosphatidyl inositol-3' kinase (PI-3K) se lie au résidu tyrosine Y751 et la phospholipase $\mathrm{C} \gamma$ (PLC $\gamma$ ) s'associe au résidu tyrosine Y785 situé dans la région carboxy-terminale de TrkA [24].

Parmi les adaptateurs, certaines molécules contiennent à la fois un domaine $\mathrm{SH} 2$ qui se lie au résidu tyrosine phosphorylé d'une protéine et deux domaines $\mathrm{SH} 3$ qui s'associent à une région riche en résidus proline d'une autre protéine. Ainsi, Grb-2 forme un complexe avec la protéine Sos par ses domaines SH3. Lors de l'activation du récepteur TrkA, le complexe cytoplasmique Grb-2/Sos est recruté à la membrane cellulaire et se lie, par l'intermédiaire du domaine $\mathrm{SH} 2$ de Grb-2, à la protéine Shc qui se trouve elle-même associée à TrkA [24, 25]. La protéine Sos est un facteur d'échange, activateur de Ras. En fait, plusieurs expériences ont montré que p21 $1^{\text {ras }}$ joue un rôle central dans la transmission des signaux engendrés par TrkA en réponse au NGF $(\mathrm{m} / \mathrm{s}$ $n^{\circ} 4$, vol. 8, p. 388) [24].

Une fois la protéine Ras activée, celle-ci se lie aux sérine/thréonine kinases de la famille Raf, à savoir Raf1 et B-Raf, provoquant ainsi leur translocation à la membrane plasmique. Les protéines Raf, alors activées, phosphorylent et activent la Mek (MAP kinase kinase) qui, à son tour, phosphoryle la MAP kinase. Cette cascade des MAP kinases entraîne alors une activation des facteurs de transcription [24, 26, 27].

Toutefois, la cascade d'événements est probablement plus complexe.
En effet, la possibilité d'une association directe entre la MAP kinase-1 (ERK1) et TrkA suggère l'existence d'une voie qui court-circuiterait la voie Ras-Raf-Mek [28]. De plus, l'interaction entre les récepteurs de la famille Trk et leur(s) ligand(s) respectif(s) induit, dans les cellules fibroblastiques NIH3T3, l'activation de signaux mitogènes qui entraînent la transformation de ces cellules. Toutefois, dans les cellules PC12, ces interactions se traduisent par une différenciation des cellules. Ces différences pourraient s'expliquer par l'existence d'une voie de transmission de signaux spécifiques de la différenciation des cellules mettant en jeu des protéines telles que la SNT(suc-associated neurotrophic factor-induced tyrosine-phosphory lated target). Cette protéine s'associe à la p13, molécule faisant partie du complexe régulateur du cycle cellulaire cdc2/cdk2 [29]. SNT est rapidement phosphorylée sur résidus tyrosine après traitement des cellules PC12 par le NGF et traitement de cultures primaires de neurones corticaux par BDNF et NT-3. Toutefois, aucune phosphorylation n'est observée lorsque les cellules PC12 sont traitées par un facteur mitogène tel que l'EGF [29]. Des expériences réalisées avec des mutants TrkA qui empêchent la signalisation par Ras mais n'altèrent pas la phosphorylation de la protéine SNT indiquent que SNT fonctionne indépendamment de Ras [30]. Enfin, des expériences récentes ont montré qu'une activation maintenue de la cascade des MAP kinases pourrait être nécessaire pour la différenciation des cellules PC12. En effet, le traitement de ces cellules avec l'EGF entraîne une activation transitoire de la MEK et une induction de la prolifération alors qu'un traitement par le NGF induit la différenciation et, dans ce cas, on observe une activation soutenue de l'activité de la MEK [31].

\section{Les multiples isoformes du récepteur TrkC: propriétés biologiques et biochimiques}

Le locus trkC a été identifié chez les mammifères par criblage d'une banque de cerveau avec une sonde codant pour le domaine tyrosine kinase du proto-oncogène trkA [15]. Le produit du gène TrkC, une glyco- 


\begin{tabular}{|c|c|c|c|c|c|c|}
\hline \multicolumn{7}{|c|}{$\begin{array}{c}\text { PROPRIÉTÉS BIOLOGIQUES DES ISOFORMES CATALYTIQUES } \\
\text { DU RÉCEPTEUR TrkC }\end{array}$} \\
\hline & \multicolumn{5}{|c|}{$\begin{array}{l}\text { Cellules } \\
\text { NIH3T3 }\end{array}$} & \multirow{2}{*}{$\begin{array}{l}\text { Cellules PC12 } \\
\text { Différenciation }\end{array}$} \\
\hline & $\begin{array}{l}\text { Liaison de } \\
\text { NT-3 }\end{array}$ & $\begin{array}{l}\text { Activation } \\
\text { par NT-3 }\end{array}$ & $\begin{array}{l}\text { Synthèse } \\
\text { d'ADN }\end{array}$ & Survie & Transformation & \\
\hline $\begin{array}{l}\text { TrkC } \\
\text { TrkC }\left(\mathrm{KI}_{14}\right) \\
\text { TrkC }\left(\mathrm{KI}_{25}\right) \\
\text { TrkC }\left(\mathrm{KI}_{39}\right)\end{array}$ & $\begin{array}{l}+ \\
+ \\
+ \\
+\end{array}$ & $\begin{array}{l}+ \\
+ \\
+ \\
+\end{array}$ & $\begin{array}{l}+ \\
+ \\
+ \\
\text { ND }\end{array}$ & $\begin{array}{l}+ \\
- \\
- \\
-\end{array}$ & $\begin{array}{l}+ \\
- \\
- \\
-\end{array}$ & $\begin{array}{l}+ \\
- \\
- \\
-\end{array}$ \\
\hline
\end{tabular}

Les diverses isoformes catalytiques du récepteur TrkC sont indiquées: récepteur dépourvu d'insertion (TrkC), récepteurs contenant une insertion de $14\left[T r k C\left(K I_{14}\right)\right], 25\left[T r k C\left(K I_{25}\right)\right]$ ou $39\left[T r k C\left(K I_{39}\right)\right]$ acides aminés dans le domaine kinase. ND: non déterminé.

protéine de $145 \mathrm{kDa}$, est un récepteur fonctionnel de forte affinité pour la neurotrophine-3 [15].

Une analyse par Northern blot révèle l'existence de plusieurs transcrits exprimés préférentiellement dans le cerveau $[15,32,33]$. trkC est largement exprimé dans le système nerveux en cours de développement et chez l'adulte, bien que le profil d'expression inclut également des cellules non neuronales. Grâce à la technique d'hybridation in situ, les transcrits $t r k \mathrm{C}$ peuvent être détectés à un stade précoce du développement embryonnaire de la souris, notamment au niveau du télencéphale et de la moelle épinière [32, 34]. Au cours du développement embryonnaire, les transcrits sont observés dans diverses régions du système nerveux central et périphérique dans lesquelles l'expression de $t r k C$ semble être corrélée de façon temporelle et spatiale à la croissance des axones vers leurs cibles [34]. De surcroît, les transcrits ont également été détectés dans le système neurovégétatif [3235 ] et plus particulièrement dans la couche musculaire externe de la paroi intestinale [34]. En fait, les études réalisées à ce jour ont montré que l'expression de $t r k C$ est corrélée à la distribution des transcrits codant pour NT-3. Cette apparente co-localisation suggère que NT-3 exerce ses effets trophiques de manière paracrine et/ou autocrine; ces effets étant responsables, au moins en partie, de la prolifération et de la différenciation des précurseurs neuronaux ainsi que de la croissance des neurones nouvellement formés.

Après la caractérisation initiale de TrkC, des isoformes possédant des insertions de taille variable dans leur domaine catalytique tyrosine kinase ont été décrites [36-38]. Ces insertions de 14, 25 et 39 acides aminés se situent en aval du site présumé d'autophosphorylation des récepteurs Trk. De telles insertions n'ont pas été trouvées dans la région correspondante d'autres récepteurs tyrosine kinases. Comme le récepteur TrkC dépourvu d'insertion, ces isoformes sont abondamment exprimées dans le système nerveux [3638]. En fait, à l'heure actuelle, aucune différence dans le profil d'expression de ces diverses isoformes n'a été mentionnée dans la littérature. Les isof ormes de TrkC contenant ces différentes insertions conservent leur capacité d'autophosphorylation en réponse à NT-3 et induisent la synthèse d'ADN dans les cellules NIH3T3 quiescentes. Toutefois, contrairement au récepteur dépourvu d'insertion, ces isoformes ne peuvent pas assurer la prolifération des cellules NIH3T3 ou la différenciation neuronale des cellules PC12 en présence de NT-3 [36-38]. L'analyse comparative des propriétés biochimiques des isoformes catalytiques a montré que seul le récepteur TrkC dépourvu d'insertion est capable de phosphoryler la PLC $\gamma$ et la PI-3 kinase [36] (Tableau I). Ces observations suggèrent que les divers effets trophiques de NT-3 dans le système nerveux des mammifères sont assurés par les multiples isoformes catalytiques du récepteur TrkC, vraisemblablement en distinguant entre différents substrats disponibles dans la cellule. Toutef ois, le rôle physiologique de ces isoformes n'est pas connu.

Une complexité plus grande des récepteurs tyrosine kinases de la famille Trk, en particulier les récepteurs TrkB et TrkC, provient de l'existence d'isoformes dépourvues de domaine catalytique tyrosine kinase. Ces isoformes possèdent des domaines extracellulaire et transmembranaire identiques à ceux des isoformes catalytiques. Toutefois, ces récepteurs ont des domaines cytoplasmiques de taille variable qui possèdent des séquences différentes dans leur région carboxy-terminale [37-39]. Le locus trkB code pour au moins un récepteur non catalytique, gp95 ${ }^{\text {ikB }}$, qui possède une très courte région cytoplasmique de 23 acides aminés dont les 11 situés dans la région carboxy-terminale diffèrent totalement des séquences présentes dans le récepteur TrkB catalytique [16]. Le locus trkC est encore plus complexe puisqu'il code pour au moins cing récepteurs non catalytiques. Un des récepteurs possède une courte région cytoplasmique de 49 acides aminés. La divergence de séquence avec gpl 45 itkc a lieu à la même position que celle décrite pour gp95 ${ }^{\imath k \mathrm{k}}$ (F. Lamballe, observation non publiée). Quatre autres isoformes non catalytiques ont été décrites dans la littérature. Leurs domaines cytoplasmiques ont des 


\section{RÉFÉRENCES}

34. Lamballe F, Smeyne RJ, Barbacid M. Developmental expression of trkC, the neurotrophin-3 receptor, in the mammalian nervous system. J Neurosci $1994 ; 14: 14-28$.

35. Chalazonitis A, Rothman TP, Chen JX, Lamballe F, Barbacid M, Gershon MD. Neurotrophin-3 induces neural crest-derived cells from fetal rat gut to develop in vitro as neurons or glia. J Neurosi 1994 ; 14: 657184.

36. Lamballe F, Tapley P, Barbacid M. trkC encodes multiple neurotrophin-3 receptors with distinct biological properties and substrate specificities. EMBO J 1993; 12 : $3083-$ 94.

37. Tsoulfas P, Soppet D, Escandon E, Tessarollo L, Mendoza-Ramirez JL, Rosenthal A, Nikolics K, Parada LF. The rat $t r k$ C locus encodes multiple neurogenic receptors that exhibit differential response to neurotrophin-3 in PC12 cells. Neuron 1993; 10: 97590.

38. Valenzuela D, Maisonpierre PC, Glass DJ, Rojas E, Nunez L, Kong Y, Gies DR, Stitt DN, Ip NY, Yancopoulos GD. Alternative forms of rat TrkC with different functional capabilities. Neuron 1993; 10: 963-74.

39. Okazawa H, Kamei M, Kanazawa I. Molecular cloning and expression of a novel truncated form of chicken $\operatorname{trkC}$. FEBS Lett 1993; 329: 171-7.

40. Dechant G, Rodriguez-Tébar A, Kolbeck $R$, Barde YA. Specific high-affinity receptors for neurotrophin-3 on sympathetic neurons. J Neurosci 1993; 13 : 2610-6.

41. Snider WD. Functions of the neurotrophins during nervous system development: what the knockouts are teaching us. Cell $1994 ; 77$ : 627-38

42. Carroll SL, Silos-Santiago I, Frese SE, Ruit KG, Milbrandt J, Snider WD. Dorsal root ganglion neurons expressing trk are selectively sensitive to NGF deprivation in utero. Neuron 1992; 9: 779-88.

43. Henderson CE, Camu W, Mettling C, $e t$ al. (13 auteurs). Neurotrophins promote motor neuron survival and are present in embryonic limb bud. Nature 1993; 363 : 266 70 .

44. Hohn A, Leibrock J, Bailey K, Barde YA. Identification and characterization of a novel member of the nerve growth factor/brain-derived neurotrophic factor family. Nature $1990 ; 344: 33941$.

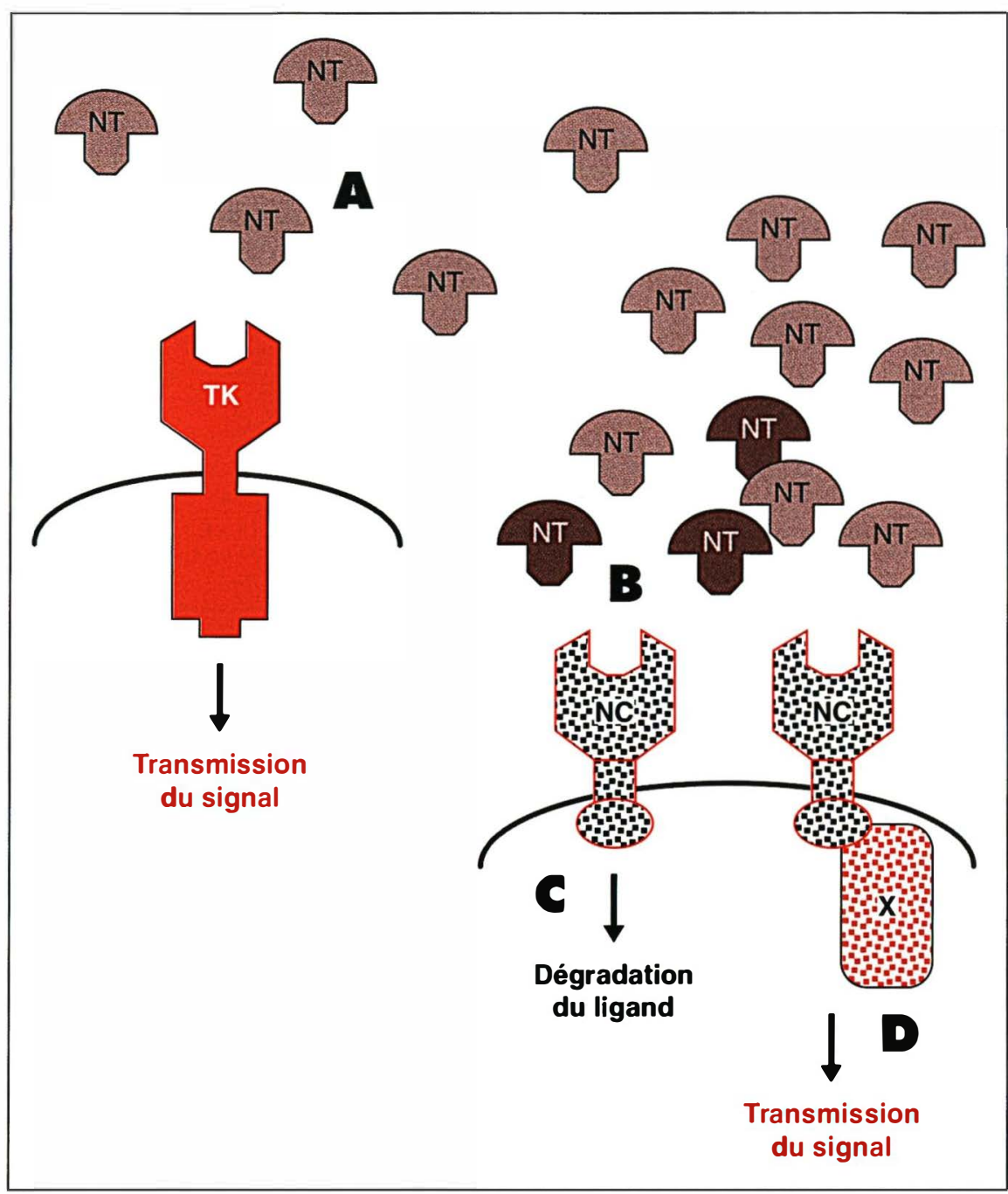

Figure 4. Rôles hypothétiques des isoformes non catalytiques des récepteurs des neurotrophines. Les récepteurs non catalytiques (NC) pourraient: (A) établir des gradients de neurotrophines (NT) et/ou présenter les neurotrophines à leurs récepteurs fonctionnels, récepteurs catalytiques tyrosine kinases (TK), situés sur d'autres cellules; (B) jouer un rôle de dominants négatifs en empêchant l'activation d'un récepteur catalytique par un ligand non préférentiel pour lequel le récepteur non catalytique a une affinité plus forte que le récepteur catalytique; (C) jouer un rôle d'épurateur du ligand qui se trouve en excès; (D) se lier et activer des protéines cytoplasmiques $(X)$ et ainsi participer à la transmission de certains signaux.

tailles variant entre 109 et 159 acides aminés; le point de divergence de séquence se situe 75 acides aminés en aval de la région transmembranaire [37-39]. Ces formes non catalytiques sont largement distribuées dans le système nerveux. Alors que les formes catalytiques des récepteurs Trk sont présentes uniquement dans les neurones, les isoformes non catalytiques de TrkB et TrkC le sont dans les neurones et dans les cellules gliales. Ces observations suggèrent plusieurs rôles pour ces récepteurs non catalytiques (figure 4). En effet, ces récepteurs pourraient établir des gradients de neurotrophines, localiser la neurotrophine à la surface des cellules et présenter ce facteur aux cellules exprimant les récepteurs catalytiques. On peut également envisager que les récepteurs non cataly- 


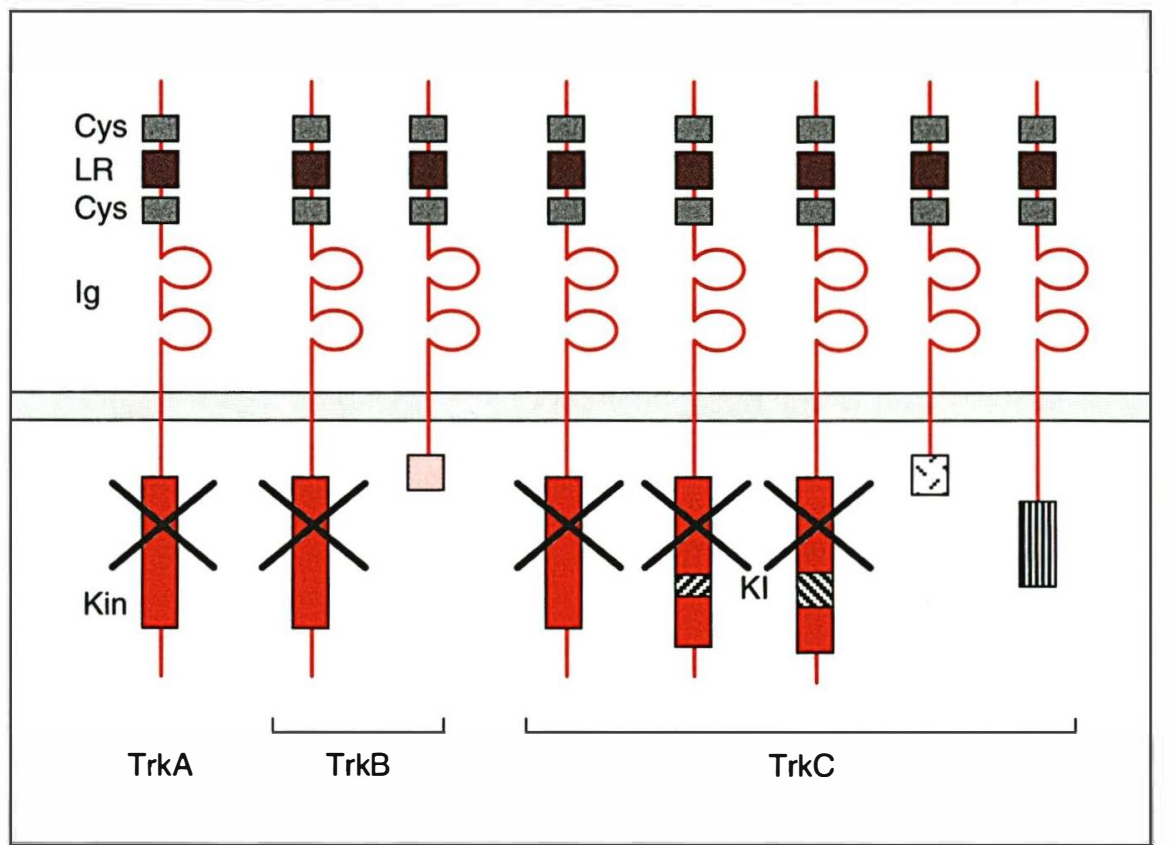

tiques, abondants à la surface de certaines cellules, neutralisent, après internalisation, le ligand lorsque l'action de ce dernier n'est plus nécessaire, par exemple après maturation des neurones [40]. Ce récepteur éliminerait alors le ligand qui se trouve en excès [16]. Troisième hypothèse: les isoformes non catalytiques pourraient agir comme dominants négatifs en empêchant l'activation d'un Trk catalytique (par exemple TrkB) par un ligand non préférentiel (comme NT-3) pour lequel le Trk non catalytique (par exemple TrkC) aurait une affinité plus forte que le récepteur Trk catalytique. En effet, le maintien d'un système sélectif d'épuration empêcherait l'accumulation de neurotrophines et, de ce fait, empêcherait l'interaction de ce facteur avec des récepteurs hétérologues. Enfin, il est également envisageable que ces récepteurs non catalytiques soient impliqués dans des voies de signalisation inconnues.

La multiplicité des isoformes catalytiques et non catalytiques est une caractéristique des récepteurs de la famille Trk. Toutefois, le rôle physiologique de ces différentes isoformes reste à élucider. Cela nous permettrait d'obtenir des informations importantes sur le mécanisme d'action des neurotrophines in vivo.

\section{Fonction des neurotrophines et de leurs récepteurs}

L'administration d'anticorps antiNGF à des souris en cours de développement a permis d'établir le rôle du NGF dans le développement et la spécificité neuronale [2]. Toutefois, cette étude ayant ses limites, d'autres approches, telles que la mutagenèse par recombinaison homologue de gènes dans les cellules souches embryonnaires, ont été adoptées pour étudier les fonctions des neurotrophines et de leurs récepteurs.

Les souris homozygotes déficientes en récepteur TrkA, TrkB ou TrkC, dénommées TrkA (-/-), TrkB (-/-) ou TrkC (-/-), ont été obtenues par délétion des séquences codant pour le domaine catalytique tyrosine kinase (figure 5).

La plupart des souris TrkA (-/-) meurent dans les trente jours qui suivent la naissance, bien que certaines atteignent l'âge adulte. Ces souris ne sont sensibles ni aux variations de température, ni à la douleur, et souffrent d'une perte sélective des neurones de petit diamètre du ganglion trigéminé et des ganglions spinaux. Les neurones sympathiques paravertébraux sont pratiquement absents à partir du dixième jour de développement embryonnaire [41]. En fait, ce phénotype est très comparable à
Figure 5. Représentation schématique des mutations nulles effectuées dans les gènes codant pour les isoformes catalytiques des récepteurs des neurotrophines. Les mutations symbolisées par les traits croisés sur le domaine Kin ont été effectuées dans les séquences codant pour les récepteurs catalytiques, laissant les isoformes non catalytiques des récepteurs TrkB et TrkC non altérées.

celui des souris exposées à des anticorps anti-NGF in utero, et en parfait accord avec la spécificité neuronale observée in vitro [42]. En outre, les souris porteuses de mutations nulles du gène codant pour le NGF, désignées souris NGF (-/-) présentent également un phénotype très semblable [41]. Ces observations montrent que les actions trophiques du NGF sont assurées par le récepteur TrkA in vivo et que cette voie de signalisation joue un rôle primordial dans le développement du système nerveux central et périphérique.

Les souris TrkB (-/-) sont déficientes en récepteur catalytique mais la mutation effectuée n'affecte pas l'isoforme non catalytique du récepteur [41] (figure 5). Les souris homozygotes pour la mutation ne têtent pas et la plupart d'entre elles meurent dans les vingt-quatre heures qui suivent la naissance. L'autopsie de ces souris révèle une forte diminution du nombre de neurones sensoriels et moteurs à la fois dans le système nerveux central et le système nerveux périphérique.

TrkB est le récepteur fonctionnel de BDNF et de NT-4/5. Or, ces deux neurotrophines assurent la survie des motoneurones et de certains types de neurones sensoriels in vitro [43]. La comparaison des phénotypes des souris TrkB (-/-) et BDNF(-/-) [41] donne une indication sur l'importance 
relative de l'action de BDNF et NT-4 sur les différents types de neurones in vivo. Les souris BDNF (-/-) et TrkB $(-/-)$ souffrent d'une diminution dramatique du nombre des neurones dans les ganglions sensoriels. Toutefois, la différence la plus frappante est que la déficience en récepteurs TrkB induit une diminution importante du nombre de motoneurones alors que les souris BDNF (-/-) sont normales à cet égard [41]. Ces observations suggèrent que, in vivo, $\mathrm{BDNF}$ est important pour la survie de certains neurones sensoriels mais pas des motoneurones. Cela laisse alors supposer que la survie des motoneurones in vivo est assurée par NT-4. Toutefois, aucune analyse du phénotype des souris déficientes en NT-4 n'a été publiée à l'heure actuelle. De plus, l'étude des phénotypes de souris déficientes à la fois en BDNF et NT-4 devrait révéler si TrkB a in vivo des fonctions supplémentaires aux actions combinées des deux ligands identifiés.

Enfin, les souris TrkC (-/-), ont été produites par délétion de séquences codant pour le domaine tyrosine kinase, laissant les isoformes non catalytiques non affectées [41] (figure 5). La plupart de ces animaux meurent dans les trois semaines qui suivent la naissance et présentent un comportement moteur anormal qui suggère un déficit dans l'information proprioceptive (information sensorielle sur la position des membres dans l'espace). Une analyse détaillée de ces souris montre qu'elles souffrent principalement d'un déficit en neurones afférents de type Ia, neurones qui innervent les muscles. Ces résultats sont en accord avec la démonstration que NT-3 assure la survie des neurones proprioceptifs in vitro [44] et avec le phénotype des souris porteuses d'une mutation nulle pour le gène codant pour NT-3 [41].

Dans toutes les expériences décrites ci-dessus, les séquences codant pour les isoformes non catalytiques des récepteurs TrkB et TrkC n'ont pas été altérées. Quel est le rôle de ces isoformes non catalytiques? Des souris porteuses de mutation dans les séquences extracellulaires des récepteurs TrkB et TrkC devraient apporter des informations.

Enfin, les résultats des croisements entre différentes souris mutantes détails anatomiques. En outre, l'étude in vitro de neurones déficients en récepteurs de la famille Trk devrait améliorer notre compréhension de la biologie cellulaire des récepteurs des neurotrophines.

\section{Perspectives thérapeutiques}

Au cours des cinq dernières années, d'importants progrès ont été réalisés dans la compréhension de la biologie et le potentiel thérapeutique des neurotrophines et leurs récepteurs: BDNF, NT-3 et NT-4/5 ont été séquencés, la structure tridimensionnelle du NGF a été résolue [3] et les produits des proto-oncogènes de la famille trk ont été identifiés comme récepteurs responsables de la transduction du signal des neurotrophines.

Des résultats expérimentaux suggérant que les neurotrophines pourraient avoir un potentiel thérapeutique dans le traitement de certains types de maladies neurodégénératives, cancer et épilepsie, une demidouzaine, ou plus, de compagnies pharmaceutiques planifient ou conduisent à l'heure actuelle des essais cliniques pour tester leur effet dans le traitement de maladies telles que la sclérose latérale amyotrophique ou les neuropathies périphériques. Toutefois, ce type de thérapie n'exclut pas l'utilisation possible d'autres facteurs trophiques. Un autre domaine important dans lequel l'utilisation de ces protéines commence à jouer un rôle est celui des maladies neurodégénératives du cerveau. La maladie d'Alzheimer est la plus répandue de ces maladies et le candidat principal pour la thérapie de cette maladie est le NGF $\left(\mathrm{m} / \mathrm{s} n^{\circ} 1\right.$, vol. 8, p. 85). Enfin, grâce aux avancées techniques concernant l'étude de la structure des gènes, la transplantation neuronale et le ciblage de certains médicaments, certains obstacles rencontrés aujourd'hui en thérapie génique seront probablement franchis dans un futur très proche $\left(\mathrm{m} / \mathrm{s} n^{\circ} 3\right.$, vol. 11, p. 475)

\section{Remerciements}

Je remercie Georges Calothy et Alain Eychene pour leur lecture critique de ce manuscrit.

\section{Summary}

The receptor tyrosine kinases of the Trk family: the high affinity neurotrophin receptors

The neurotrophins of the NGF family are involved in regulating neuronal differentiation, growth and survival. Their action is mediated by the members of the Trk family of receptors tyrosine kinases. However, they also bind to another receptor, $\mathrm{p} 75^{\mathrm{LNGFR}}$, whose role remains controversial. The Trk receptors, predominantly expressed in the nervous system, are rather unique in that they have multiple isoforms (some non catalytic and others with inserts in the catalytic kinase domain) that might have distinct physiological roles. Analysis of mice carrying null mutations for the neurotrophins and their receptors should provide valuable informations for our understanding of the function of these factors and their possible use in the treatment of various types of neurological diseases.

\section{TIRÉS À PART}

\section{F. Lamballe}

